\title{
Application of Artificial Intelligence Techniques for Detection of Cracks-A Review
}

\author{
D. N. Thatoi, A. A. Ojha, A. Bhanjadeo, R. Mahapatra, S. Sahoo, and S. Mohapatra
}

\begin{abstract}
Detection of cracks using vibration analysis employs different algorithms out of which artificial intelligence (AI) techniques is drawing a lot of attention of the engineers and scientists. This paper presents a comprehensive review of various AI techniques for determining the location, size and depth of cracks in different structures. The techniques mainly consist of fuzzy interference technique, neural network (NN), genetic algorithm (GA), hybrid models and other optimization algorithms.
\end{abstract}

Index Terms-Genetic, hybrid models, neural network, swarm optimization.

\section{INTRODUCTION}

Condition monitoring and fault detection through vibration analysis applying a pool of analytical and experimental techniques is of continuous attention of researchers. The effectiveness and applicability of each technique has both advantages and limitations. There are various methods being employed for the detection of cracks such as Finite Element Method (FEM), Wavelet analysis, Experimental and Numerical methods, Artificial Intelligence (AI) techniques, other optimization algorithm methods such as Particle Swarm Optimization (PSO) algorithm, Ant Colony Optimization technique (ACO) and Bee Colony Optimization (BCO) algorithm.

Progression in the fault diagnosis process has led to automated feature extraction and subsequent fault detection and identification. In this regard, artificial techniques and optimization algorithm techniques are gaining much more importance as compared to other methods because of their ease of application, reliable, accurate and precise results. Numerous attempts have been made in the past to employ artificial intelligence techniques such as fuzzy analysis, neural network analysis, and genetic algorithm and hybrid models. Saravanan [1] employed fuzzy interference technique in order to determine the condition of an inaccessible gear in an operating machine. Buezas [2] used genetic algorithm optimization method in order to deal with crack detection in structural elements. Rosales [3] used neural network technique for the detection of cracks in beam like structure. Hybrid models are also being employed such as neuro-fuzzy, genetic-fuzzy, neuro-genetic and neural, genetic and fuzzy combined together. Wang [4] employed neuro-fuzzy network to forecast damage propagation trend in

Manuscript received July 27, 2012; revised September 29, 2012.

The authors are with the S'O'A University, Department of Mechanical Engineering, I. T. E. R., Bhubaneswar, Odisha (e-mail massthatoi@gmail.com, aam4u123@gmail.com, auroprit182@gmail.com, mahapatra.rishav@gmail.com, sarthakmohapatra90@gmail.com). rotary machinery and to provide an alarm before a fault reaches critical levels. Zheng [5] used genetic fuzzy algorithm for structural health monitoring of composite laminated beams. Maity [6] used hybrid neuro-genetic algorithm for clamped-free beam and plane frame.A novel strategy using Particle Swarm Optimization (PSO) was being employed by Begambre [7] for structural damage identification. Inspired by the collective behavior of real ant colonies, Ant Colony Optimization (ACO) algorithm was introduced. It is a probabilistic technique for solving computational problems which can be employed to find best paths through graphs. Kang [8] introduced a hybrid simplex artificial bee colony algorithm (HSABCA) for inverse analysis problems.

\section{Genetic Algorithm FOR CRACK Detection}

Genetic algorithm belongs to a larger class of evolutionary algorithm. It is a search technique used to find an approximate solution to optimization and search problems. Baghmisheh [9] proposed a fault diagnosis method based on GA for a cracked structure. An analytical model of a damaged cantilever beam was considered and natural frequencies were calculated. The crack was modeled as a torsional spring and using Hamilton's principle the equation of motion of the cracked beam was determined. This model was used for analysis of different kinds of damaged structures. Buezas [2] applied genetic algorithms to detect crack on damaged structural elements up-to level three detection. They optimized the objective function which was derived from the dynamics of the studied body. First of all they performed crack detection on a beam using computational experiments and then used physical experiment to validate the methodology. The computational studies were performed to find the optimum GA values.

\section{FUZZY INTERFERENCE TECHNIQUE FOR CRACK DETECTION}

For dealing with uncertainty, fuzzy logic is a precise approach. Fuzzy inference can be described as an approach that interprets the values in an input vector and based on some set of rules it assigns the values to an output vector. Rules are inputs for building a fuzzy inference. Saravanan [1] used this technique as decision tree for selecting best statistical features to discriminate the fault conditions of the gear box from the signals extracted. Fabio [10] proposed a general methodological approach for the fuzzy reliability analysis of structural systems in case of reinforced and pre stressed concrete structures. Fuzzy criterion is used to model all the uncertainties in which the model is defined through a band of 
values within minimum and maximum extremes. The reliability problem at the load level with reference to several serviceability and ultimate limit states has been formulated and the strategic planning of the solution process is done using Genetic algorithm. The potentiality of a reinforced prestressed beam able to take both material and geometrical non-linearity into account is taken into consideration and exploited and particular attention is paid to both the solution of the optimization process, based on a genetic algorithm, and the structural analysis techniques.

\section{NEURAL NETWORK ANALYSIS FOR DETECTION OF CRACKS}

Neural network (NN) has been successfully implemented in detection of faults in various cases. A neural network (NN), in the case of artificial neurons called artificial neural network (ANN) is an interconnected group of natural or artificial neurons that uses a mathematical or computational model for information processing based on a connection approach to computation. It consists of potentially large number of simple processing elements known as nodes or neurons. Each neuron simply computes a nonlinear weighted sum of its inputs, and transmits the result over its outgoing connections to other neurons. Liu [11] proposed an inverse analysis using the back-propagation $\mathrm{NN}$ and computational mechanics combining the finite element method with boundary integral equation has been proposed and applied to the detection and sizing of cracks. The computational mechanics can be used to create relatively inexpensive simulated inspection A-scan data for various cracks as long as the function of transducer pulse is known. The classification and the identification of cracks were reasonable using this technique. Elshafey [12] proposed a random decrement technique which is used to extract the free decay of the structure which outlines the use of the NN technique in the identification of a damage index for an offshore structure using its free decay response. The method presented can be used routinely to discover any shape change of the damage index. With the help of an infield inspection, the exact location and magnitude of damage can be determined.

\section{HYBRID TECHNIQUES}

\section{A. Genetic-Fuzzy Analysis}

The genetic fuzzy system is primarily used for detection of cracks and its effect on the structures. The primary advantage of genetic fuzzy over traditional fuzzy system is that when the number of parameters of input and output function of a system increases, GF system provides an easy solution for the model as compared to the fuzzy network which gets plagiarized when complexity increases. This work was validated by Pawar [13] in his work for damage detection of beams and blades of a helicopter rotor. They considered a non-uniform beam and BO-105 hinge less rotor blade of a helicopter for testing purposes. The data obtained were excellent in the domain of frequency and mode shape of the rotor and the beam.

\section{B. Neuro-Genetic System}

Here, genetic algorithm is used to encode the parameters of neural networks as a string of properties of the network. This can locate the neighborhood of the optimal solution more efficiently as compared to other conventional methods. Suh [14] found a method to identify the location and depth of crack on a faulty structure by the help of hybrid neuro-genetic method. First of all a feed forward multi-layered NN having back-propagation was used to learn the input i.e. location and depth of crack and a structural eigen frequencies i.e. output relation of the structural system. Then GA is applied to this neural network to identify the location and depth of crack thereby minimizing the difference from the measured frequencies.

\section{Neuro-Fuzzy Technique}

Neuro-fuzzy technique was proposed by J. S. R. Jang and this hybridization resulted in a hybrid intelligent system which synergizes the two techniques by combining the human-like reasoning style of fuzzy systems with the learning and connectionist structure of neural networks. Wang [15] developed a machine fault prognostic system by applying this technique and tested it against faulty gears. They also took into account the previous works on shaft misalignment and gear pit damage. The results obtained proved that the machine fault prognostic system was able to detect the damage location and its effect precisely and efficiently.

\section{Neural-Genetic-Fuzzy Analysis}

Keeping an eye on the flexibility of various artificial intelligence techniques, Saridakis [16] applied neural networks, genetic algorithms and fuzzy logic for the identification of cracks in shafts. First, a model was devised which was used to find the rotational angle of the crack. Next an efficient objective function was defined using fuzzy logic and more intelligence was added using genetic algorithm. This was used to find the characteristics of the crack using artificial neural network.

\section{OTHER OPTIMIZATION ALGORITHMS}

\section{A. Particle Swarm Optimization}

Particle swarm optimization was originally found to simulate the problems of social behavior i.e. to study the behavior of society or interaction between members of same species. Kennedy [17] introduced this technique to solve nonlinear functions. The common topologies associated with PSO are p-best, l-best and g-best. The value which records the trajectory of the problem domain is called p-best. The best value obtained by a particle in the neighborhood of its journey is called l-best. Now if the particle incorporates all the elements or particles as its topological neighbors then it is called g-best. The advanced or hybrid form of PSO based on simplex algorithm was proposed by Begambre [7] for crack identification in damaged structures particularly a ten bar truss and cracked beam.

\section{B. Ant Colony Optimization}

It is a member of swarm intelligence proposed initially by Dorigo [18] in his research works. The technique so proposed was a probabilistic approach of finding various paths using graphs. In simple sense it has been described as the natural world of ants. Ants move scatterly for food and upon successful attempt of finding the food, they leave their pheromone on their return. Pheromone triggers a response 
from other members of the ants who are a part of that society to follow the same path as followed by their predecessors. When the followed path fails to yield desired results for the ants they change course and look set to find another path which is much shorter and density of pheromone is high. $\mathrm{Hu}$ [19] proposed an improve form of ant colony optimization to solve continuous optimization problems and named it as continuous orthogonal ant colony. Basically this new phenomenon enabled the ants to search their domain faster and efficiently.

\section{Bee Colony Optimization Algorithm}

Bee's optimization is a search algorithm developed by Karaboga [20]. It draws its inspiration from the food searching prowess or behavior of a small group of honeybees. As the search is random in direction this method is useful in solving functional optimization as well as combinational optimization problems (finding a suitable object from a group of objects). Moradi [21] used the bee's algorithm for identification of cracks in beam structures. In their work they concluded that in case of experimental verification BA was far superior to PSO. It was also found that in comparision to other techniques it is much simpler, accurate and reliable for crack detection.

\section{RESULTS AND DISCUSSION}

The genetic algorithm clearly demonstrated its capability to create good approximate solutions in complex optimization problems. It is very attractive in comparison with classical methods since it does not require a space within the whole space solution. This approach is capable of detecting small crack location and depth with small errors. Arbitrary shaped bodies can be handled with a nonlinear elastic approach. Different shaped cracks irrespective of its size, its corresponding model and its simulation can easily be tackled.

The fault identification based on fuzzy logic technique shows its great potentiality to classify and identify machinery faults. This model yielded a significant enhancement in the prediction of shear strength without neglecting principles of mechanics governing shear failure in concrete beams. Fuzzy approach is an effective approach to handle complex structural systems. It is a precise approach for dealing with uncertainty.

Artificial intelligence involving neural network made a breakthrough performance in detecting cracks in composite materials. It also worked in detecting the damage index of cases involving free decay of structures. It is also clearly evident from the facts that combining the individual artificial techniques resulted in an improved form called as hybrid techniques which were found to be more reliable and efficient As observed, fuzzy system gets plagiarized when complexity of system increases. In this case the use of genetic fuzzy hybrid technique was found to be more useful and efficient. Similarly the combining of neuro-genetic system was found to be more efficient than conventional systems in finding the optimal solution of the neighboring damage element. Neuro-fuzzy combination technique too came into the picture for detection of cracks in damaged structures and its effect on the system. Moreover, not undermining the flexibility of these techniques a combination of all the AI techniques resulted in the development of genetic fuzzy- neural network which was effective in even determining the orientation and rotational angle of the crack.

Barring these AI techniques it has been observed that some techniques were derived taking the help of natural characteristics. The development of PSO, ACO and BCO algorithms may seem to be very old optimization techniques but they beautifully relate the social characteristics or behaviour of each particle of a faulty system.

\section{CONCLUSION}

This paper presented various techniques and methodologies for comprehensive analysis of cracks in different structures. From the analysis carried out, it is found out that among the various techniques artificial intelligence techniques are efficient as compared to others for the determination of location and intensity of the crack in a structure. Moreover, it is also observed that hybrid techniques are more efficient in terms of fault finding and reliable in case of complex systems than conventional AI techniques. AI technique will continue to remain one of the favorite analytical tools to extract features automatically in fault diagnosis due to its precise, reliable and low cost solution nature.

\section{REFERENCES}

[1] N. Saravanan, S. Cholairajan and K. I. Ramachandran, Expert Systems with Applications, vol. 36, pp. 3119-3135, 2009.

[2] F. S. Buezas, M. B. Rosales, and C. P. Filipich, Engineering Fracture Mechanics, vol. 78, pp. 695-712, 2011.

[3] M. B. Rosales, C. P. Filipich, and F. S. Buezas, Engineering Structures, vol. 31, pp. 2257-2264, 2009.

[4] W. Q. Wang, M. F. Golnaraghi, and F. Ismail, Mechanical Systems and Signal Processing, vol. 18, pp. 813-831, 2004.

[5] S.-J. Zheng, Z.-Q. Li and H.-T. Wang, Expert Systems with Applications, vol. 38, pp. 11837-11842, 2011.

[6] B. Sahoo and D. Maity, Applied Soft Computing, vol. 7, pp. 89-104, 2007

[7] O. Begambre and J. E. Laier, Advances in Engineering Software, vol. 40, pp. 883-891, 2009

[8] F. Kang, J. Li, and Q. Xu, Computers and Structures, vol. 87, pp. 861-870, 2009.

[9] M. T. V. Baghmisheh, M. Peimani, M. H. Sadeghiand, M. Mohammad, Ettefagh: Applied Soft Computing, vol. 8, pp. 1150-1160, 2008.

[10] F. Biondini, F. Bontempi, and P. Giorgio, Malerba: Computers and Structures, vol. 82, pp. 1033-1052, 2004.

[11] S. W. Liua, J. H. Huanga, J. C. Sungb and C. C. Leeb, Comput. Methods Appl. Mech. Engrg. vol. 191, pp. 2831-2845, 2002.

[12] A. A. Elshafey, M. R. Haddara, and H. Marzouk, Marine Structures, vol. 23, pp. 131-145, 2010.

[13] P. M. Pawar and R. Ganguli, Comput. Methods Appl. Mech. Engrg.vol. 192, pp. 2031-2057, 2003.

[14] M. W. Suh, M. B. Shim, and M. Y. Kim, J. Sound Vibration, vol. 238, no. 4, pp. $617-635,2000$.

[15] W. Q. Wang, M. F. Golnaraghib, and F. Ismail, Mechanical Systems and Signal Processing, vol. 18, pp. 813-831, 2004.

[16] K. M. Saridakis, A. C. Chasalevris, C. A. Papadopoulos, and A. J. Dentsoras, Computers and Structures, vol. 86, pp. 1318-1338, 2008.

[17] J. Kennedy and R. Eberhart, "Particle swarm optimization," Proceedings of IEEE International Conference on Neural Networks IV, pp. 1942-1948, 1995.

[18] M. Dorigo, "Optimization, learning and natural algorithm," Phd Thesis, Politecnico di Milano, Italy, 1992.

[19] X. Hu, J. Zhang, and Y. Li, Journal of Computer Science and Technology, vol. 23, no. 1, pp. 2-18, 2008

[20] D. Karaboga and B. Basturk, "An artificial bee colony (ABC) algorithm for numeric function optimization," IEEE Swarm Intelligence Symposium, Indianapolis, Indiana, USA, 2006.

[21] S. Moradi, P. Razi, and L. Fatahi, Computers and Structures, vol. 89, pp. 2169-2175, 2011. 